\title{
Pain related and overall morbidity with TRUS guided prostate biopsy - a prospective study
}

\author{
Sarvpreet S. Ubee, Rajendra R. Marri, Shalom J. Srirangam \\ Department of Urology, East Lancashire Hospitals NHS Trust, Blackburn, United Kingdom
}

\section{ABSTRACT}

Objective: To assess analgesia requirement after trans-rectal ultrasound guided prostate biopsy(TRUSBx) for appropriate counselling.

Materials and Methods: Prospectively, successive patients undergoing TRUSBx between July 2009 and November 2011 were given questionnaires prior to procedure. Standard 12-core TRUSBx under peri-prostatic block (10 mL of $1 \%$ lidocaine) and antibiotic prophylaxis (oral ciprofloxacin, intravenous gentamicin and metronidazole suppository) were performed. Pain perception was assessed using a Visual Analogue Score (VAS).

Results: Mean (range) age of the 405 patients was 67.3 years (48-88). Mean VAS during the procedure was 2.93 and 2.20 on reaching home. Mean maximum VAS for the cohort on day 1 and day 2 were 1.27 and 0.7 respectively. 140 (35\%) were independent with some or minimal discomfort. 14 patients required assistance for some of their basic daily needs. 9 patients (2.2\%) were hospitalised due to sepsis. 131 patients (32.4\%) required additional oral analgesia following TRUSBx on days 0,1 and 2 . These patients were generally younger with a mean age for this group of 63.6 years (46-88). The difference in the mean age between those self-medicating and not was not statistically significant ( $p$ > 0.005). This group had mean VAS during the procedure of 4 and when patients reached home was 3.5. Mean maximum VAS on day 1 and 2 was 2.1 and 1.3 respectively. 11 patients required assistance from another adult.

Conclusion: A third of patients required self-medicated analgesia post-procedure. Age alone cannot be used as a criterion to identify patients who will subsequently require analgesia post-procedure, but a higher VAS during the procedure may be indicative. These patients must be counselled appropriately.

\section{ARTICLE INFO}

\section{Key words:}

Ultrasound, High-Intensity

Focused, Transrectal; Prostate;

Biopsy; Analgesia; Hematuria

Int Braz J Urol. 2013; 39: 671-4

Submitted for publication: January 30, 2013

Accepted after revision: August 15, 2013

\section{INTRODUCTION}

Transrectal ultrasound guided prostate biopsy (TRUSBx) is one of the more commonly carried out procedures on a day case basis. In the immediate absence of a conclusive, readily available and safe new alternative technique for prostate cancer detection, the number of patients undergoing TRUSBx will continue to rise due to increasing awareness about prostate cancer. Thou- gh TRUSBx equipment has improved with smaller probes and superior image quality, the procedure still provokes considerable anxiety for patients. Foremost in the patient's mind is the possibility of a diagnosis of prostate cancer. Added to this concern, is the discomfort associated with the procedure and the apprehension of possible complications. Periprostatic nerve block at the time of TRUSBx is now well established in reducing pain associated with the procedure (1-4). 
The counselling of patients for TRUSBx within most centres in the United Kingdom is consistent with the evidence based information provided by the British Association of Urological Surgeons (5). Much is known about the discomfort associated with the procedure but data for analgesia requirements post-procedure are inconsistent. The objective of this study was to prospectively assess the analgesia requirement and biopsy related morbidity following TRUSBx, which would enable better counselling of patients.

\section{MATERIALS AND METHODS}

A prospective study was planned and approval granted by the Research and Audit department of the hospital trust after review of the protocol and the questionnaire. Successive patients undergoing TRUSBx between July 2009 and November 2011 were enrolled. Patients were given questionnaires at the time of pre-procedure counselling and instructed to record responses immediately after the procedure and at various points for two days post biopsy.

Patients underwent a standard 12-core TRUSBx under local anaesthetic infiltration peri-prostatic nerves with $10 \mathrm{~mL}$ of $1 \%$ lidocaine (without adrenaline). All biopsies were carried out by either a trainee or an experienced urologist while performing trans-rectal ultrasound with the patient in a lateral decubitus position. All patients received a 3 day course of ciprofloxacin and single doses of gentamicin with metronidazole. Thirty minutes prior to the TRUSBx, patients were administered one gram of ciprofloxacin orally along with $160 \mathrm{mg}$ of intravenous gentamicin. At the completion of the procedure, metronidazole suppository was inserted and the patients were sent home with a three day course of oral ciprofloxacin. Pain perception was measured on a visual analogue scale (VAS 1-10) at different times of the day during and after the procedure (day 0). On days 1 and 2 post procedure patients reported the maximum and minimum VAS score. Ability to perform normal daily activities, use of self-medicated painkillers over this three day period was reported. Patients also recorded episodes of haematuria, haematospermia, urinary retention, urinary tract infection and episodes of post biopsy urinary sepsis were also noted from hospital database.

\section{RESULTS}

Over the study period of 28 months, 448 patients underwent TRUSBx. We received questionnaires from 405 patients giving us a response rate of $90.4 \%$. The mean (range) age of the cohort was 67.3 years (48-88). The mean (range) VAS during the procedure for the whole cohort $(n=405)$ was $2.93(0-10)$ and when patients reached home it was $2.20(0-10)$ on day 0 . The mean (range) maximum VAS for the cohort on days 1 and 2 was $1.27(0-10)$ and $0.7(0-8)$ respectively.

The majority (63\%) were fully independent after the procedure while 35\% had some or minimal discomfort. 14 patients required assistance for some of their daily needs. In total, 267 patients (74\%) reported no complications after TRUSBx, while, haematuria (22.4\%), haemospermia $(11.8 \%)$, urinary retention $(2.7 \%)$ and sepsis requiring hospital admission (2.2\%) were reported in the remainder. These all were grade I/II on morbidity as per the Clavien classification of surgical complications (6).

Additional oral analgesia following TRUSBx was required by 131 patients (32.4\%) on days 0,1 and 2 . These patients were generally younger with a mean age for this group of 63.6 years (46-88). The difference in the mean age between the group of patients self-medicating and not self-medicating was not statistically significant. For this group of patients the mean VAS during the procedure was 4 and when patients reached home, it was 3.5. VAS was 5 or more in $40 \%$ of the patients. Mean maximum VAS on days 1 and 2 was 2.1 and 1.3 , respectively. The mean VAS was higher in these patients compared to those did not self medicate analgesia (Table-1). Eleven patients of the 131 required assistance for some of their daily needs. The mean age for these 11 patients was 69 years and a mean (range) VAS of 4 (1-8) during the TRUSBx.

\section{DISCUSSION}

TRUSBx seems to be well tolerated by majority of men and the emphasis has always been to reduce the associated pain and discomfort during the TRUSBx. One study of 104 men found that 
Table 1 - Comparative VAS between the patients who did and did not self medicate analgesia post procedure.

\begin{tabular}{lcc}
\hline Mean VAS & $\begin{array}{c}\text { No post-procedure analgesia } \\
(\mathrm{n}=271 / 405)\end{array}$ & $\begin{array}{c}\text { Post procedure analgesia } \\
(\mathrm{n}=131 / 405)\end{array}$ \\
\hline During Procedure & 2.4 & 4.0 \\
Day 0 (Night) & 1.35 & 3.5 \\
Day 1 Maximum & 0.8 & 2.1 \\
Day 2 Maximum & 0.4 & 1.3 \\
\hline
\end{tabular}

$24 \%$ experienced moderate to extreme pain when between 4 and 8 biopsies were performed (7), while others demonstrated that $19 \%$ of men would not wish to undergo the procedure again without some form of analgesia (8).

Naughton et al. found a significant increase in pain recall at 2 weeks following the procedure, which persisted after 4 weeks (9). This fact has implications for future patient compliance when repeat prostate sampling is necessary. In our study the patients who self-medicated analgesia had an overall higher VAS compared to the remainder. It is therefore important to identify this group of patients as post-procedural pain needs to be well controlled in order to improve patient care.

Cadaveric studies have shown that the neuroanatomical pathway originates from the inferior hypogastric plexus located at the tip of the seminal vesicles and passes between the prostate and rectum on the inferolateral border of the prostate (10). These neural fibers transmit visceral sensation from the prostatic capsule to the spinal cord, explaining the discomfort associated with transrectal prostate biopsy. As the neurovascular bundles are in close proximity to the rectal wall, they are easily accessible by an ultrasound-guided needle and the predictable course of these nerves renders the injection of an anesthetic feasible, thus providing significantly improved pain control as proven by various studies (1-4). The post-procedural pain may be associated with the stretching of the prostate capsule due to the bleeding from the glandular tissue thus contributing to the dis- comfort following the TRUSBx. This bleeding may be more aggressive in some than others thereby making them more uncomfortable and requiring analgesia post procedure.

The ProBE trial has shown that although 85\% of the patients undergoing TRUSBx described no or mild procedure associated pain during TRUSBx but within 35 days pain was an undesirable effect in $44 \%$ of which $7 \%$ found it to be a moderate or serious problem (11).

It can be argued that some degree of pain is expected with any minor day case procedure, but it is important to note from our study that patients, though a very small proportion, found the pain to be debilitating enough to ask assistance from another individual for some of their daily needs. The post-procedural period for this small number of patients was not complicated by any significant complications associated with TRUSBX.

\section{CONCLUSIONS}

Though only in a small number, this routine office based procedure can have temporary debilitating effect of these patients requiring assistance for their daily needs. Higher VAS during the procedure can be an indicator for identifying these patients along with those who require additional analgesia for increased pain post-TRUSBX. We feel it is important that this group of patients undergoing TRUSBx should take regular oral analgesia at least for the next 2 days following 
the procedure. This should form part of the counselling process as for some of the patients the only access to seek medical attention would be emergency services.

\section{ABBREVIATIONS}

TRUSBX $=$ Trans-rectal ultrasound guided prostate biopsy

VAS $=$ Visual analogue score

\section{CONFLICT OF INTEREST}

None declared.

\section{REFERENCES}

1. Alavi AS, Soloway MS, Vaidya A, Lynne CM, Gheiler EL: Local anesthesia for ultrasound guided prostate biopsy: a prospective randomized trial comparing 2 methods. J Urol. 2001; 166: 1343-5.

2. Pareek G, Armenakas NA, Fracchia JA: Periprostatic nerve blockade for transrectal ultrasound guided biopsy of the prostate: a randomized, double-blind, placebocontrolled study. J Urol. 2001; 166: 894-7.

3. Berger AP, Frauscher F, Halpern EJ, Spranger R, Steiner H, Bartsch G, et al.: Periprostatic administration of local anesthesia during transrectal ultrasound-guided biopsy of the prostate: a randomized, double-blind, placebo-controlled study. Urology. 2003; 61: 585-8.

4. Lynn NN, Collins GN, Brown SC, O’Reilly PH: Periprostatic nerve block gives better analgesia for prostatic biopsy. BJU Int. 2002; 90: 424-6.
5. The british association of urological surgeons: Available at: www.baus.org.uk/Resources/BAUS/Documents/PDF\%20 Documents/Patient\%20information/TRUSP.pdf. Accessed March 2012.

6. Dindo D, Demartines N, Clavien PA: Classification of surgical complications: a new proposal with evaluation in a cohort of 6336 patients and results of a survey. Ann Surg. 2004; 240: 205-13.

7. Crundwell MC, Cooke PW, Wallace DM: Patients' tolerance of transrectal ultrasound-guided prostatic biopsy: an audit of 104 cases. BJU Int. 1999; 83: 792-5.

8. Irani J, Fournier F, Bon D, Gremmo E, Doré B, Aubert J: Patient tolerance of transrectal ultrasound-guided biopsy of the prostate. Br J Urol. 1997; 79: 608-10.

9. Naughton CK, Ornstein DK, Smith DS, Catalona WJ: Pain and morbidity of transrectal ultrasound guided prostate biopsy: a prospective randomized trial of 6 versus 12 cores. J Urol. 2000; 163: 168-71.

10. Hollabaugh RS Jr, Dmochowski RR, Steiner MS: Neuroanatomy of the male rhabdosphincter. Urology. 1997; 49: 426-34.

11. Rosario DJ, Lane JA, Metcalfe C, Donovan JL, Doble A, Goodwin L, et al.: Short term outcomes of prostate biopsy in men tested for cancer by prostate specific antigen: prospective evaluation withinProtecT study. BMJ. 2012; 344: d7894.

Correspondence address: Sarvpreet S Ubee, MD Department of Urology East Lancashire Hospitals NHS Trust Haslingden road, Blackburn, BB1 3HH, UK Telephone: + 04412 828-05076 E-mail: sarv21@hotmail.com 